\title{
T2 Candida Panel: A Game Changer in Diagnosis of Fungal Infections
}

\author{
Devesh $\mathrm{N} \mathrm{Joshi}^{1}$, Bhaskar Shenoy ${ }^{2}$
}

\begin{abstract}
In invasive fungal infections, early initiation of treatment within the day of symptom onset is related with a significantly reduced mortality. Early initiation of treatment and specifically treatment initiation within the day of symptom onset and blood culture draw is related to a significantly reduced mortality. However, automated blood culture methodologies, which are routinely used for the diagnosis of candidemia, take up to 2-5 days to grow and lead to a crucial delay in treatment initiation. Therefore, a highly sensitive, specific, and rapid diagnostic method is expected to allow early initiation of antifungal therapy and subsequently improve outcomes. Rapid diagnostic methods for fungal infections are long-awaited and are expected to improve outcomes through early initiation of targeted antifungal therapy. T2 Candida panel is a novel qualitative diagnostic platform that was recently approved by the US Food and Drug Administration (FDA) for diagnosis of candidemia with a mean time to species identification of $<5$ hours. Technological advances in the field of nanotechnology coupled with the proven applications of magnetic resonance have recently presented a rapid, fully automated, qualitative, sensitive, and specific diagnostic platform, the "T2 Candida panel". The introduction of this technology in diagnostic algorithms will increase the cost per patient tested, but it is expected to provide an economically self-supporting policy if savings from shorter hospital stays and termination of excess empiric antifungal treatment are taken into account. Expected benefits in terms of morbidity, mortality, and costs remain to be confirmed in clinical practice. The T2 Magnetic Resonance (T2MR) assay can detect and speciate the five most common Candida spp.; namely, Candida albicans, Candida glabrata, Candida parapsilosis, Candida tropicalis, and Candida krusei, in approximately 5 hours. The five detectable Candida spp. are responsible for $>95 \%$ of the total Candida infections. T2 Magnetic Resonance demonstrated a sensitivity and specificity of 91.1 and $98.1 \%$, respectively. The utility of T2MR in candidemia depends on the prevalence of the disease in each clinical setting. In intensive care units and other high-prevalence settings, the incorporation of T2MR in diagnostic algorithms is very appealing. T2 Magnetic Resonance is expected to allow timely initiation of antifungal therapy and help with antifungal stewardship. In low-prevalence settings, the positive predictive value of T2MR might not be enough to justify the initiation of antifungal treatment in itself.
\end{abstract}

Keywords: Candida, Investigation, T2 Candida panel.

Pediatric Infectious Disease (2022): 10.5005/jp-journals-10081-1333

\section{INTRODUCTION}

Consequent to the increase of immunocompromised patients, an increase in the incidence of invasive fungal infections has been documented. ${ }^{1}$ Early initiation of treatment and treatment initiation within the day of symptom onset and blood culture draw is related with a significantly reduced mortality. ${ }^{2,3}$ However, automated blood culture methodologies, which are routinely used for the diagnosis of candidemia, take up to 2-5 days to grow and lead to a crucial delay in treatment initiation.

Therefore, a highly sensitive, specific, and rapid diagnostic method is expected to allow early initiation of antifungal therapy and subsequently improve outcomes. ${ }^{4}$ Moreover, a rapid diagnostic method has the potential to reduce the administration of empiric antifungal treatment to patients who test negative, reducing this way the adverse effects of antifungal therapy, ${ }^{5}$ as well as the antimicrobial pressure that is associated with the development of resistance to antifungal agents. ${ }^{6-8}$

Rapid diagnostic methods for fungal infections are longawaited and are expected to improve outcomes through the early initiation of targeted antifungal therapy. T2 Candida panel is a novel qualitative diagnostic platform that was recently approved by the US Food and Drug Administration (FDA) for diagnosis of candidemia with a mean time to species identification of $<5$ hours. Technological advances in the field of nanotechnology coupled with the proven applications of magnetic resonance have recently presented a rapid, fully automated, qualitative, sensitive, and

\footnotetext{
1,2Department of Paediatrics, Manipal Hospital, Bengaluru, Karnataka, India
}

Corresponding Author: Bhaskar Shenoy, Department of Paediatrics, Manipal Hospital, Bengaluru, Karnataka, India, Phone: +91 9845036174, e-mail: bshenoy@gmail.com

How to cite this article: Joshi DN, Shenoy B. T2 Candida Panel: A Game Changer in Diagnosis of Fungal Infections. Pediatr Inf Dis 2022; 4(1):24-25.

Source of support: Nil

Conflict of interest: None

specific diagnostic platform, the "T2 Candida panel". ${ }^{9} \mathrm{~T} 2$ Candida panel combines nuclear magnetic resonance and PCR molecular assays to directly detect and identify Candida spp. from whole blood samples. ${ }^{9}$

The introduction of this technology in diagnostic algorithms will increase the cost per patient tested, but it is expected to provide an economically self-supporting policy if savings from shorter hospital stays and termination of excess empiric antifungal treatment are taken into account. ${ }^{7,9}$ Expected benefits in terms of morbidity, mortality, and costs remain to be confirmed in clinical practice. This new technology may represent a paradigm shift in the field of infectious diseases diagnostics.

Invasive candidiasis is a common healthcare-associated infection with a high mortality rate that can exceed $60 \%$ in cases 
of septic shock. Blood culture performance is far from ideal, due to a long time of positivity and suppression by antifungal agents. The T2 Magnetic Resonance (T2MR) assay can detect and speciate the five most common Candida spp.; namely, Candida albicans, Candida glabrata, Candida parapsilosis, Candida tropicalis, and Candida krusei, in approximately 5 hours. The five detectable Candida spp. are responsible for $>95 \%$ of the total Candida infections. ${ }^{10}$ It should be noted that the T2 Candida panel does not estimate the resistance profile of the isolated Candida spp. Using quantified spiked samples, the limit of detection of T2 Candida panel was estimated to be $1 \mathrm{CFU} / \mathrm{mL}$ for C. tropicalis and C. krusei, $2 \mathrm{CFU} / \mathrm{mL}$ for C. albicans and C. glabrata, and $3 \mathrm{CFU} / \mathrm{mL}$ for $C$. parapsilosis. In a multicenter clinical trial that included both a prospective and a contrived arm to represent the full range of clinically relevant concentrations of Candida spp., T2MR demonstrated a sensitivity and specificity of 91.1 and $98.1 \%$, respectively. The utility of T2MR in candidemia depends on the prevalence of the disease in each clinical setting. In intensive care units and other high-prevalence settings, the incorporation of T2MR in diagnostic algorithms is very appealing. T2 Magnetic Resonance is expected to allow timely initiation of antifungal therapy and help with antifungal stewardship. In lowprevalence settings, the positive predictive value of T2MR might not be enough to justify the initiation of antifungal treatment in itself. The performance of T2MR has not been studied in cases of deep-seated candidiasis. Despite some promising evidence in published clinical trials, further studies are needed to determine the performance of T2MR in invasive candidiasis without candidemia.

The T2 Candida panel can identify five Candida spp. and the following three results can be reported: ${ }^{5}$

- Candida albicans/Candida tropicalis.

- Candida parapsilosis.

- Candida glabrata/Candida krusei.

\section{CONCLUSION}

Upcoming T2 Magnetic Resonance for Fungal Diagnosis panel can be a gamechanger in the management of invasive fungal infections. It can identify five important Candida species in 3 hours with $100 \%$ sensitivity and $98 \%$ specificity and helps decide antifungal medication early. Although it cannot replace old age gold standard investigation-blood culture for drug sensitivity pattern, but it is cost-effective in the management of Candida infection and will help in reducing morbidity and mortality due to invasive candidemia.

T2 Magnetic Resonance represents a highly promising molecular diagnostic method that allows the rapid, accurate, and species-specific diagnosis of candidemia. The positive T2MR results should be interpreted in the context of the expected prevalence of the disease in the specific clinical setting. We should note the paucity of data regarding the cases with culture-negative invasive candidiasis and the evolving everyday clinical experience with this new technology. Future studies remain to determine the performance of T2MR in patients diagnosed with deep-seated infections by following those patients with T2MR, blood cultures, and fungal markers.

\section{References}

1. Singh T, Kashyap AK, Ahluwalia G, et al. Epidemiology of fungal infections in critical care setting of a tertiary care teaching hospital in North India: a prospective surveillance study. Mortality 2014;1:19-25.

2. Bassetti M, Giacobbe DR, Vena A, et al. Incidence and outcome of invasive candidiasis in intensive care units (ICUs) in Europe: results of the EUCANDICU project. Critical Care 2019;23(1):1-7. DOI: 10.1186/ s13054-019-2497-3.

3. Avni T, Leibovici L, Paul M. PCR diagnosis of invasive candidiasis: systematic review and meta-analysis. J Clin Microbiol 2011;49(2):665670. DOI: 10.1128/JCM.01602-10.

4. Neely LA, Audeh M, Phung NA, et al. T2 magnetic resonance enables nanoparticle-mediated rapid detection of candidemia in whole blood. Sci Translat Med 2013;5(182):182ra54. DOI: 10.1126/ scitransImed.3005377.

5. Zervou FN, Zacharioudakis IM, Kurpewski J, et al. T2 magnetic resonance for fungal diagnosis. In Human fungal pathogen identification. New York, USA: Humana Press; 2017. pp. 305-319.

6. Beyda ND, Alam MJ, Garey KW. Comparison of the T2Dx instrument with T2 Candida assay and automated blood culture in the detection of Candida species using seeded blood samples. Diagnos Microbiol Infect Dis 2013;77(4):324-326. DOI: 10.1016/j.diagmicrobio.2013. 07.007 .

7. Mylonakis E, Clancy CJ, Ostrosky-Zeichner L, et al. T2 magnetic resonance assay for the rapid diagnosis of candidemia in whole blood: a clinical trial. Clin Infect Dis 2015;60(6):892-899. DOI: 10.1093/ cid/ciu959.

8. Pappas PG, Kauffman CA, Andes D, et al. Clinical practice guidelines for the management of candidiasis: 2009 update by the Infectious Diseases Society of America. Clin Infect Dis 2009;48(5):503. DOI: 10.1086/596757.

9. Bilir SP, Ferrufino CP, Pfaller MA, et al. The economic impact of rapid Candida species identification by T2 Candida among high-risk patients. Fut Microb 2015;10(7):1133-1144. DOI: 10.2217/fmb.15.29.

10. Pfaller MA, Jones RN, Castanheira M. Regional data analysis of Candida non-albicans strains collected in United States medical sites over a 6-year period, 2006-2011. Mycoses 2014;57(10):602-611. DOI: 10.1111/myc.12206. 